\title{
Reliability analysis for preventive maintenance based on classical and Bayesian semi-parametric degradation approaches using locomotive wheel-sets as a case study
}

\author{
Jing Lin ${ }^{\mathrm{a}, \mathrm{b}, *}$, Julio Pulido ${ }^{\mathrm{c}}$, Matthias Asplund ${ }^{\mathrm{a}, \mathrm{d}}$ \\ a Division of Operation and Maintenance Engineering, Luleå University of Technology, 97187 Luleå, Sweden \\ ${ }^{\mathrm{b}}$ Luleå Railway Research Centre, 97187 Luleå, Sweden \\ ${ }^{\mathrm{c}}$ ReliaSoft Corporation, Tucson, AZ 85710-6703, USA \\ ${ }^{\mathrm{d}}$ Swedish Transport Administration, 97125 Luleå, Sweden
}

\section{A R T I C L E I N F O}

\section{Article history:}

Received 18 August 2013

Received in revised form

7 October 2014

Accepted 8 October 2014

Available online 28 October 2014

Keywords:

Reliability

Preventive maintenance

Degradation models

Accelerate life testing

Bayesian approach

\begin{abstract}
A B S T R A C T
This paper undertakes a general reliability study using both classical and Bayesian semi-parametric degradation approaches. The goal is to illustrate how degradation data can be modelled and analysed to flexibly determine reliability to support preventive maintenance strategy making, based on a general data-driven framework. With the proposed classical approach, both accelerated life tests (ALT) and design of experiments (DOE) technology are used to determine how each critical factor affects the prediction of performance. With the Bayesian semi-parametric approach, a piecewise constant hazard regression model is used to establish the lifetime using degradation data. Gamma frailties are included to explore the influence of unobserved covariates within the same group. Ideally, results from the classical and Bayesian approaches will complement each other. To demonstrate these approaches, this paper considers a case study of locomotive wheel-set reliability. The degradation data are prepared by considering an Exponential and a Power degradation path separately. The results show that both classical and Bayesian semi-parametric approaches are useful tools to analyse degradation data and can, therefore, support a company in decision making for preventive maintenance. The approach can be applied to other technical problems (e.g. other industries, other components).
\end{abstract}

(c) 2014 Elsevier Ltd. All rights reserved.

\section{Introduction}

Estimating the failure-time distribution or long-term performance of components of high reliability products is particularly difficult. Many modern products are designed to operate without failure for a long time, for example, train wheel-sets. Thus, few units will fail or significantly degrade in a test of practical length at normal use conditions.

The service life of train wheel-sets can be significantly reduced due to failure or damage, leading to excessive cost and accelerated deterioration, a point which has received considerable attention in recent literature. In order to monitor the performance of wheel-sets and make replacements in a timely fashion, the railway industry uses both preventive and predictive maintenance. By predicting the wear [14,2,29], fatigue [1,17], tribological aspects [5], and failures [31],

\footnotetext{
* Corresponding author.

E-mail addresses: janet.lin@ltu.se (J. Lin), Julio.pulido@reliasoft.com (J. Pulido), Matthias.asplund@ltu.se (M. Asplund).
}

the industry can design strategies for different types of preventive maintenance (re-profiling, lubrication, etc.) for various periods (days, months, seasons, running distance, etc.). Software dedicated to predicting wear rate has also been proposed [22]. Finally, condition monitoring data have been studied with a view to increasing the wheel-sets' lifetime [25,8,28,21].

Preventive maintenance (PM) actions are usually performed at predetermined points in time to keep the reliability of the system at a desired level [9]. One common preventive maintenance strategy (used in the case study) is re-profiling wheel-sets after they run a certain distance. Re-profiling affects the wheel's diameter; once the diameter is reduced to a pre-specified length, the wheel-set is replaced by a new one. Seeking to optimise this maintenance strategy, researchers have examined wheel-set degradation data to determine wheel-set reliability and failure distribution. However, in previous studies, some researchers have noticed that the wheel-sets' different installed positions may influence the results. To avoid this possibility, Freitas et al. [11] only consider those wheels on the left side of a specified axle and on certain specified cars, arguing that "the degradation of a 
given wheel might be associated with its position on a given car". Yang and Letourneau [31] suggest certain attributes, including a wheel's installed position (right or left), might influence its wear rate, but they do not provide case studies. Palo [21] conclude that "different wheel positions in a bogie show significantly different force signatures". Recently, to solve the combined problem of small data samples and incomplete datasets whilst simultaneously considering the influence of several covariates, Lin et al. [16] have explored the influence of locomotive wheels' positioning on reliability with Bayesian parametric models. Their results indicate that the particular bogie in which the wheel is mounted has more influence on its lifetime than does the axle or which side it is on. As this paper is an extension of the study in Lin et al. [16], in addition to the locomotive, we only use the bogie as a main influence factor.

To achieve complementary results, we perform a reliability study using both classical and Bayesian semi-parametric frameworks and propose a general data-driven framework. In the case study, we explore the impact of a locomotive wheel-set's position on its service lifetime to predict its other reliability characteristics. The goal is to illustrate how a wheel-set's degradation data can be modelled and analysed using both classical and Bayesian approaches in order to flexibly determine reliability for preventive maintenance strategy making based on the proposed frameworks. Notably, this data-driven method [33] can be extended to other applications.

The remainder of the paper is organised as follows. Section 2 presents the degradation models using a classical approach. We use both accelerated life tests (ALT) and design of experiments (DOE) technology to determine how each critical factor affects the prediction of performance. Section 3 presents the piecewise constant hazard regression model with gamma frailties. In the proposed model, a discrete-time martingale process is considered as a prior process for the baseline hazard rate. It adopts a Markov Chain Monte Carlo (MCMC) computational scheme. Section 4 proposes a general data-driven framework as a summary and Section 5 describes the case study of the wheel-sets on two locomotives in a heavy haul cargo train, using both exponential and power degradation assumptions, and discusses maintenance strategies for optimisation. Finally, Section 6 offers conclusions and comments.

\section{Classical approach}

In this section, we use a general log linear (GLL) life stress relationship to analyse the degradation data with accelerated life tests (ALT), considering lifetime data from a specific degradation path. Then, using the specific degradation model, we perform a two factor full factorial design of experiments analysis.

\subsection{Accelerated life testing}

As discussed above, many modern products are designed to operate without failure for a long time. Under such conditions, few units will fail or significantly degrade in a test of practical length at normal use conditions. For this reason, accelerated life tests (ALTs) are widely used in manufacturing industries, particularly to obtain timely information on the reliability of product components and materials. Generally, information from tests at high stress levels of accelerating variables (e.g., use rate, temperature, voltage, or pressure) is extrapolated, through a physically reasonable statistical model (e.g. Eiren, Arrhenius, Inverse Power Law), to obtain estimates of life or long-term performance at lower and normal use conditions. ALT results are used in design-for reliability processes to assess or demonstrate component and subsystem reliability, certify components, detect failure modes, compare manufacturers, and so forth. ALTs have become increasingly important because of rapidly changing technologies, more complicated products with more components, and higher customer expectations of better reliability.

In some reliability studies, it is possible to measure degradation directly over time, either continuously or at specific points in time. In most reliability testing applications, degradation data, if available, can have important practical advantages [15]: particularly in applications where few or no failures are expected, they can provide considerably more reliability information than would be available from traditional censored failure-time data. Accelerated tests are commonly used to obtain reliability test information more quickly Direct observation of the degradation process (e.g., tire wear) may allow direct modelling of the failure-causing mechanism, providing more credible and precise reliability estimates and a valid basis for extrapolation. Modelling degradation of the performance output of a component or subsystem (e.g., voltage or power) may be useful, but modelling could be complicated or difficult because the output may be affected, albeit unknowingly, by more than one physical/ chemical failure-causing process.

Once we obtain the projected failures values for each degradation model, we can carry out an accelerated life analysis using some critical factors as stress factors. The analysis can be performed using a general log linear (GLL) life stress relationship with a Weibull probability function, modelled as:

$L(X)=e^{\left(\alpha_{0}+\sum_{i=1}^{m} \alpha_{i} X_{i}\right)}$

This model (2.1) can also be expressed as an exponential model, with life as a function of the stress vector $X$, where $X$ is a vector of $n$ stressors [18]. For this analysis, we consider stress applications of the model and a logarithmic transformation on $X$, such that $X=\ln (V)$ where $V$ is the specific stress. This transformation generates an inverse power model life stress relationship, as shown below for each stress factor:

$L(V)=\frac{1}{K V^{n}}$

\subsection{Design of experiments analysis}

Design of experiment (DOE) analysis has been widely applied to improving product performance [10], as for example, in numerous recent reliability studies: DOE makes it possible to explore multiple stressors in an efficient way for reliability improvement considering the no fault found (NFF) phenomenon [26]; a two-level full factorial DOE may identify the reliability of the reverse link as the performance metric of real-time traffic [12]; a DOE based parameter tuning is proposed to provide an estimation of turbine power plant availability [3]; a two factorial DOE analysis based on the cost associated with maintenance and replacement activities and reliability characteristic parameters is used to determine the optimal preventive maintenance and replacement schedules in repairable and maintainable systems [19]; a two-level, three-factor experiment is used to collect human performance data under different mustering conditions [20].

Reliability DOE (R-DOE) can be used to identify factors affecting product life and can also be used to optimise design variables to improve product reliability [23]. It is fairly similar to the analysis of other designed experiments except the response is the life of the product in the respective units (e.g., for an automobile component, the units of life may be miles, for a mechanical component this may be cycles, and for a pharmaceutical product, this may be months or years). Note: In Section 5.3, we perform a two factor full factorial R-DOE analysis. 


\section{Bayesian semi-parametric approach}

Most reliability studies are implemented under the assumption that individual lifetimes are independent identically distributed (i.i.d). At times, however, Cox proportional hazard $(\mathrm{CPH})$ models cannot be used because of the dependence of data within a group. For instance, because they have the same operating conditions, the wheel-sets mounted on a particular locomotive may be dependent. In a different context, some data may come from multiple records which actually belong to wheel-sets installed in the same position but on another locomotive. Modelling dependence in multivariate survival data has received considerable attention in cases where the datasets may come from subjects of the same group which are related to each other in some fashion. A key development in modelling such data is to consider frailty models, in which the data are conditionally independent. When frailties are considered, the dependence within subgroups can be considered an unknown and unobservable risk factor (or explanatory variable) of the hazard function. In this section, we consider a gamma shared frailty, first discussed by Clayton [4] and later developed by Sahu et al. [24], to explore the influence of unobserved covariates. In addition, since semi-parametric Bayesian methods offer a more general modelling strategy with fewer assumptions, we adopt the piecewise constant hazard model to establish the distribution of the lifetime. The applied hazard function is sometimes called a piecewise exponential model (PEM; [7]); it is convenient because it can accommodate various shapes of the baseline hazard over the intervals. This model can also be viewed as a nonparametric ALT model [32].

\subsection{Piecewise constant hazard regression model}

The piecewise constant hazard model is one of the most convenient and popular semi-parametric models in survival analysis. We begin by denoting the $j$ th individual in the $i$ th group as having lifetime $t_{i j}$, where $i=1, \ldots, n$ and $j=1, \ldots, m_{i}$. Divide the time axis into intervals $0<s_{1}<s_{2}<\cdots<s_{k}<\infty$, where $s_{k}>t_{i j}$, thereby obtaining $k$ intervals $\left(0, s_{1}\right],\left(s_{1}, s_{2}\right], \ldots\left(s_{k-1}, s_{k}\right]$. Suppose the $j$ th individual in the $i$ th group has a constant baseline hazard $h_{0}\left(t_{i j}\right)=\lambda_{k}$ as in the $k$ th interval, where $t_{i j} \in I_{k}=\left(s_{k-1}, s_{k}\right]$. Then, the hazard rate function for the piecewise constant hazard model can be written as

$h_{0}\left(t_{i j}\right)=\lambda_{k}, \quad t_{i j} \in I_{k}$

Eq. (3.1) is sometimes referred to as a piecewise exponential model (PEM); it can accommodate various shapes of the baseline hazard over the intervals.

Ref. [7] summarise studies on how to divide the time axis into kintervals. However, using common cutpoints simplifies both the notation and the ease of understanding the ideas [6]. In this paper, we discuss the choice of $k$ in the case study.

Suppose $\mathbf{x}_{i}=\left(x_{1 i}, \cdots x_{p i}\right)^{\prime}$ denotes the covariate vector for the individuals in the ithgroup, and $\boldsymbol{\beta}$ is the regression parameter. Therefore, the regression model with the piecewise constant hazard rate can be written as

$h\left(t_{i j}\right)= \begin{cases}\lambda_{1} \exp \left(\mathbf{x}_{\mathrm{ij}}^{\prime} \boldsymbol{\beta}\right) & 0<t_{i j} \leq s_{1} \\ \lambda_{2} \exp \left(\mathbf{x}_{\mathrm{ij}}^{\prime} \boldsymbol{\beta}\right) & s_{1}<t_{i j} \leq s_{2} \\ \vdots & \vdots \\ \lambda_{k} \exp \left(\mathbf{x}_{\mathrm{ij}}^{\prime} \boldsymbol{\beta}\right) & s_{k-1}<t_{i j} \leq s_{k}\end{cases}$

Its corresponding probability density function $f\left(t_{i j}\right)$, cumulative distribution function $F\left(t_{i j}\right)$, reliability function $R\left(t_{i j}\right)$, together with the cumulative hazard rate $\Lambda\left(t_{i j}\right)$, can now be achieved.

\subsection{Gamma shared frailty model}

Frailty models were first considered by Clayton [4] to handle multivariate survival data. In these models, the event times are conditionally independent according to a given frailty factor, an individual random effect. As discussed by Sahu et al. [24], the models formulate different variabilities and come from two distinct sources. The first source is natural variability, explained by the hazard function; the second is variability common to individuals of the same group or variability common to several events of an individual, explained by the frailty.

Assume the hazard function for the $j$ th individual in the $i$ th group is

$h_{i j}(t)=h_{0}(t) \exp \left(\mu_{i}+\mathbf{x}_{\mathrm{ij}}^{\prime} \boldsymbol{\beta}\right)$

In Eq. (3.3), $\mu_{i}$ represents the frailty parameter for the $i$ th group. By denoting $\omega_{i}=\exp \left(\mu_{i}\right)$, the equation can be written as

$h_{i j}(t)=h_{0}(t) \omega_{i} \exp \left(\mathbf{x}_{\mathbf{i j}}^{\prime} \boldsymbol{\beta}\right)$

Eq. (3.3) is an additive frailty model, and Eq. (3.4) is a multiplicative frailty model. In both equations, $\mu_{i}$ and $\omega_{i}$ are shared by the individuals in the same group; they are thus referred to as sharedfrailty models and are actually extensions of the $\mathrm{CPH}$ model.

To this point, discussions of frailty models have focused on the choices of the form of the baseline hazard function and the form of the frailty's distribution. Wienke [30] explores different frailty models from both univariate and multivariate perspectives. In this paper, we consider the piecewise constant hazard rate due to its flexibility, as well as the gamma shared frailty model, the most popular model for frailty.

From Eq. (3.4), suppose the frailty parameters $\omega_{i}$ are independent and identically distributed (i.i.d) for each group and follow a gamma distribution, denoted by $G a\left(\kappa^{-1}, \kappa^{-1}\right)$. In this case, the probability density function can be written as

$f\left(\omega_{i}\right)=\frac{\left(\kappa^{-1}\right)^{\kappa^{-1}}}{\Gamma\left(\kappa^{-1}\right)} \cdot \omega_{i}^{\kappa^{-1}-1} \exp \left(-\kappa^{-1} \omega_{i}\right)$

In Eq. (3.5), the mean value of $\omega_{i}$ is 1 , where $\kappa$ is the unknown variance of $\omega_{i}$ s. Greater values of $\kappa$ signify a closer positive relationship between the subjects of the same group, as well as greater heterogeneity among groups. Furthermore, as $\omega_{i}>1$, the failures for the individuals in the corresponding group will appear earlier than if $\omega_{i}=1$; in other words, as $\omega_{i}<1$, their predicted lifetimes will be greater than those found in the independent models.

Suppose $\boldsymbol{\omega}=\left(\omega_{1}, \omega_{2}, \cdots, \omega_{n}\right)^{\prime} ;$ then

$\pi(\boldsymbol{\omega} \mid \kappa) \propto \prod_{i=1}^{n} \omega_{i}^{\kappa^{-1}-1} \exp \left(-\kappa^{-1} \omega_{i}\right)$

\subsection{Discrete-time martingale process for baseline hazard rate}

Based on the above discussion (Eqs. (3.2), (3.4), and (3.5)), the piecewise constant hazard model with gamma shared frailties can be written as:

$h\left(t_{i j}\right)= \begin{cases}\lambda_{1} \omega_{i} \exp \left(\mathbf{x}_{\mathbf{i j}}^{\prime} \boldsymbol{\beta}\right) & 0<t_{i j} \leq s_{1} \\ \lambda_{2} \omega_{i} \exp \left(\mathbf{x}_{\mathbf{i j}}^{\prime} \boldsymbol{\beta}\right) & s_{1}<t_{i j} \leq s_{2} \\ \vdots & \vdots \\ \lambda_{k} \omega_{i} \exp \left(\mathbf{x}_{\mathbf{i j}}^{\prime} \boldsymbol{\beta}\right) & s_{k-1}<t_{i j} \leq s_{k}\end{cases}$

In Eq. (3.7), $\omega_{i} \sim G a\left(\kappa^{-1}, \kappa^{-1}\right)$. 


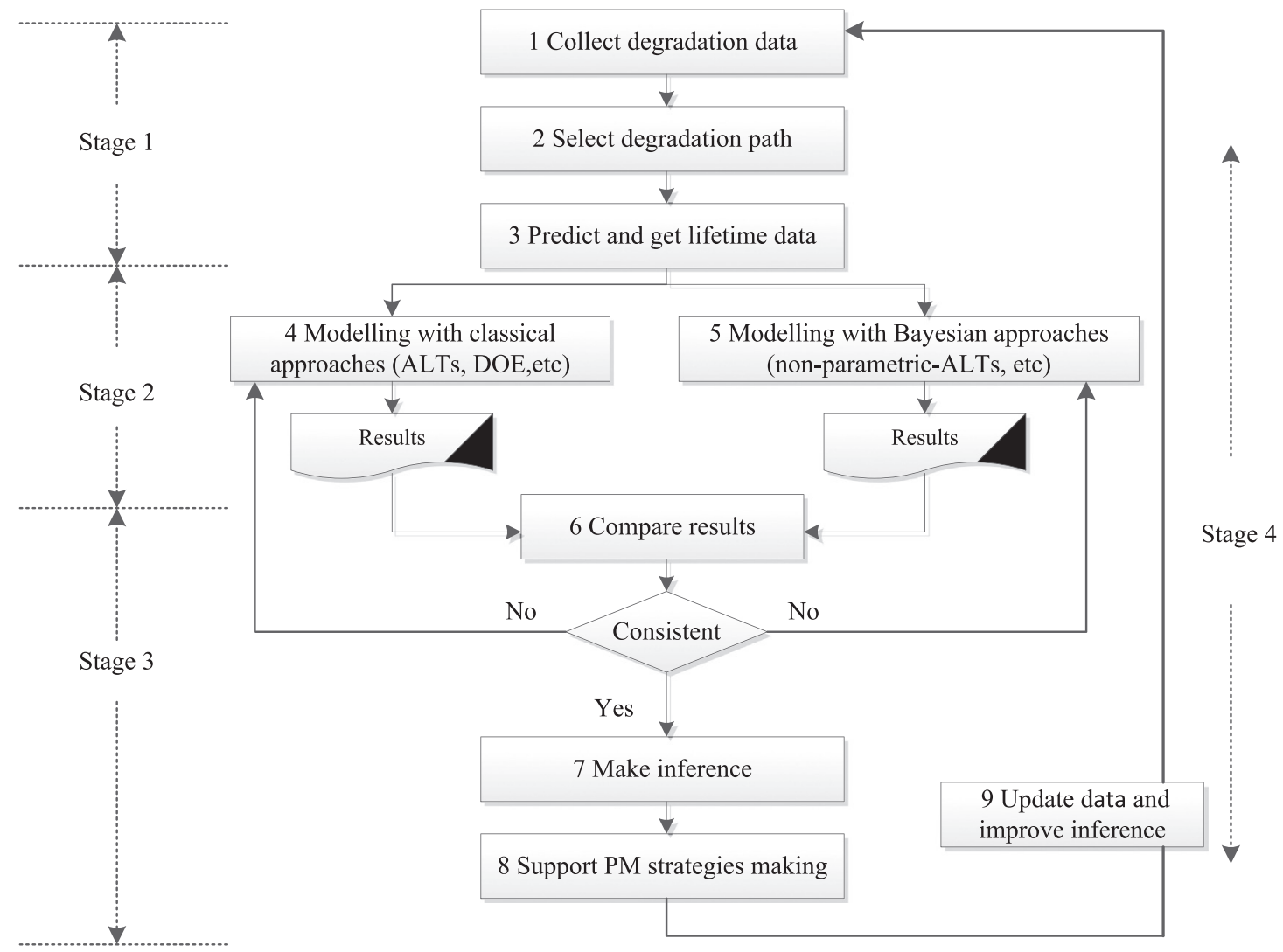

Fig. 4.1. A general data-driven framework.

To analyse the baseline hazard rate $\lambda_{k}$, a common choice is to construct an independent incremental process, e.g., the Gamma process, the Beta process, or the Dirichlet process. However, as pointed out by Ibrahim et al. [13], in many applications, prior information is often available on the smoothness of the hazard rather than the actual baseline hazard itself. In addition, given the same covariates, the ratio of marginal hazards at the nearby timepoints is approximately equal to the ratio of the baseline hazards at these points. In such situations, correlated prior processes for the baseline hazard can be more suitable. Such models, for instance, the discrete-time martingale process for the baseline hazard rate $\lambda_{k}$, are discussed by Sahu et al. [24].

Given $\left(\lambda_{1}, \lambda_{2}, \cdots, \lambda_{k-1}\right)$, we specify that

$\lambda_{k} \mid \lambda_{1}, \lambda_{2}, \cdots, \lambda_{k-1} \sim G a\left(\alpha_{k}, \frac{\alpha_{k}}{\lambda_{k-1}}\right)$

Let $\lambda_{0}=1$. In Eq. (3.8), the parameter $\alpha_{k}$ represents the smoothness for the prior information. If $\alpha_{k}=0$, then $\lambda_{k}$ and $\lambda_{k-1}$ are independent. As $\alpha_{k} \rightarrow \infty$, the baseline hazard is the same in the nearby intervals. In addition, the Martingale $\lambda_{k}$ 's expected value at any time point is the same, and

$E\left(\lambda_{k} \mid \lambda_{1}, \lambda_{2}, \cdots, \lambda_{k-1}\right)=\lambda_{k-1}$

Eq. (3.9) shows that given specified historical information $\left(\lambda_{1}, \lambda_{2}, \cdots, \lambda_{k-1}\right)$, the expected value of $\lambda_{k}$ is fixed.

\subsection{Bayesian semi-parametric model using MCMC}

In reliability analysis, the lifetime data are usually incomplete, and only a portion of the individual lifetimes are known. Right-censored data are often called Type I censoring, and the corresponding likelihood construction problem is extensively studied in the literature. Suppose the $j$ th individual in the $i$ th group has lifetime $T_{i j}$ and censoring time $L_{i j}$. The observed lifetimet $t_{i j}=\min \left(T_{i j}, L_{i j}\right)$; therefore, the exact lifetime $T_{i j}$ will be observed only if $T_{i j} \leq L_{i j}$. In addition, the lifetime data involving right censoring can be represented by $n$ pairs of random variables $\left(t_{i j}, v_{i j}\right)$, where $v_{i j}=1$ if $T_{i j} \leq L_{i j}$ and $v_{i j}=0$ if $T_{i j}>L_{i j}$. This means that $v_{i j}$ indicates whether lifetime $T_{i j}$ is censored or not. The likelihood function is deduced as

$L(t)=\prod_{i=1}^{n} \prod_{j=1}^{m_{i}}\left[f\left(t_{i j}\right)\right]^{v_{i j}} R\left(t_{i j}\right)^{1-v_{i j}}$

In the above piecewise constant hazard model, we denote $g_{i j}$ as $t_{i j} \in\left(s_{g_{i j}}, s_{g_{i j}+1}\right)=I_{g_{i j}+1}$ and the model's dataset as $D=(\boldsymbol{\omega}, \mathbf{t}, \mathbf{X}, \mathbf{v})$. Following Eqs. (3.7)-(3.10), the complete likelihood function $L(\boldsymbol{\beta}, \lambda \mid D)$ for the individuals for the ith group in $k$ intervals can be written as

$$
\begin{aligned}
& \prod_{i=1}^{n} \prod_{j=1}^{m_{i}}\left\{\left[\prod_{k=1}^{g_{i j}} \exp \left(-\lambda_{k} \omega_{i} \exp \left(\mathbf{x}_{\mathbf{i j}}^{\prime} \boldsymbol{\beta}\right)\left(s_{k}-s_{k-1}\right)\right]\right.\right. \\
& \left.\quad \times\left(\lambda_{g_{i j}+1} \omega_{i} \exp \left(\mathbf{x}_{\mathbf{i j}}^{\prime} \boldsymbol{\beta}\right)\right)^{v_{i j}} \times \exp \left[-\lambda_{g_{i j}+1} \omega_{i} \exp \left(\mathbf{x}_{\mathbf{i j}}^{\prime} \boldsymbol{\beta}\right)\left(t_{i j}-s_{g_{i j}}\right)\right]\right\}
\end{aligned}
$$

Let $\pi(\cdot)$ denote the prior or posterior distributions for the parameters. Following Eqs. (3.6) and (3.11), the joint posterior distribution $\pi\left(\omega_{i} \mid \boldsymbol{\beta}, \lambda, D\right)$ for gamma frailties $\omega_{i}$ can be written as

$$
\begin{aligned}
& \pi\left(\omega_{i} \mid \boldsymbol{\beta}, \lambda, D\right) \propto L(\boldsymbol{\beta}, \lambda \mid D) \times \pi(\boldsymbol{\omega} \mid \kappa) \\
& \quad \propto \kappa^{-1}+\sum_{j=1}^{m_{i}} v_{i j}-1 \\
& \propto \omega_{i} \exp \left\{-\left(\kappa^{-1}+\left[\sum_{j=1}^{m_{i}} \exp \left(\mathbf{x}_{\mathbf{i j}}^{\prime} \boldsymbol{\beta}\right)\right]\right)\right.
\end{aligned}
$$




$$
\begin{aligned}
& \left.\times\left(\sum_{k=1}^{g_{i j}} \lambda_{k}\left(s_{k}-s_{k-1}\right)+\lambda_{g_{i j}+1}\left(t_{i j}-s_{g_{i j}}\right)\right)\right\} \\
\sim & G a\left\{\kappa^{-1}+\sum_{j=1}^{m_{i}} v_{i j}, \kappa^{-1}+\left[\sum_{j=1}^{m_{i}} \exp \left(\mathbf{x}_{\mathbf{i j}}^{\prime} \boldsymbol{\beta}\right)\right]\left(\sum_{k=1}^{g_{i j}} \lambda_{k}\left(s_{k}-s_{k-1}\right)\right.\right. \\
& \left.\left.+\lambda_{g_{i j}+1}\left(t_{i j}-s_{g_{i j}}\right)\right)\right\}
\end{aligned}
$$

Eq. (3.12) shows that the full conditional density of each $\omega_{i}$ is a gamma distribution. Similarly, the full conditional density of $\kappa^{-1}$ and $\boldsymbol{\beta}$ can be given by

$$
\begin{gathered}
\pi\left(\kappa^{-1} \mid \boldsymbol{\beta}, \boldsymbol{\omega}, \lambda, D\right) \propto \prod_{i=1}^{n} \omega_{i}^{\kappa^{-1}-1}\left(\kappa^{-1}\right)^{-n \kappa^{-1}} \\
\times \frac{\exp \left(-\kappa^{-1} \sum_{i=1}^{n} \omega_{i}\right)}{\left[\Gamma\left(\kappa^{-1}\right)\right]^{n}} \cdot \pi\left(\kappa^{-1}\right)
\end{gathered}
$$

$$
\begin{gathered}
\pi\left(\boldsymbol{\beta} \mid \kappa^{-1}, \boldsymbol{\omega}, \boldsymbol{\lambda}, D\right) \propto \exp \left\{\sum_{i=1}^{n} \sum_{j=1}^{m_{i}} v_{i j} \mathbf{x}_{\mathbf{i j}}^{\prime} \boldsymbol{\beta}-\sum_{i=1}^{n} \sum_{m=1}^{n_{i}} \exp \left(\mathbf{x}_{\mathbf{i j}}^{\prime} \boldsymbol{\beta}\right) \omega_{i}\right. \\
\left.\times\left[\sum_{k=1}^{g_{i j}} \lambda_{k}\left(s_{k}-s_{k-1}\right)+\lambda_{g_{i j}+1}\left(t_{i j}-s_{g_{i j}}\right)\right]\right\} \times \pi(\boldsymbol{\beta})
\end{gathered}
$$

Let $R_{k}=\left\{(i, j) ; t_{i j}>s_{k}\right\}$ denote the risk set at $s_{k}$ and $D_{k}=R_{k-1}-$ $R_{k}$; let $d_{k}$ denote the failure individuals in the interval $I_{k}$.

\begin{tabular}{|c|c|c|c|c|c|c|c|c|c|c|c|c|}
\hline \multirow[t]{3}{*}{ Distance (km) } & \multicolumn{12}{|c|}{ Degradation (mm) } \\
\hline & \multicolumn{6}{|c|}{ Bogie I } & \multicolumn{6}{|c|}{ Bogie II } \\
\hline & 1 & 2 & 3 & 4 & 5 & 6 & 7 & 8 & 9 & 10 & 11 & 12 \\
\hline 106613 & 13.08 & 13.19 & 12.11 & 12.12 & 12.99 & 13.04 & 13.02 & 13.01 & 11.94 & 12.01 & 13.01 & 13.16 \\
\hline 144207 & 27.11 & 27.07 & 23.01 & 22.86 & 25.03 & 25.09 & 24.09 & 24.12 & 23.95 & 24.06 & 26.56 & 26.55 \\
\hline 191468 & 38.95 & 38.94 & 39.11 & 39.06 & 39.15 & 39.17 & 35.95 & 35.95 & 35.88 & 35.93 & 36.24 & 36.04 \\
\hline 272697 & 70.6 & 70.53 & 69.94 & 69.87 & 69.9 & 69.9 & 79.7 & 79.73 & 79.73 & 79.74 & 79.59 & 79.76 \\
\hline 309426 & 85.05 & 85.07 & 85.09 & 85.12 & 85.26 & 85.27 & 1 & 1 & 1 & 1 & 82.87 & 83.77 \\
\hline
\end{tabular}
Let $\pi\left(\lambda_{k} \mid \lambda^{(-k)}\right.$.)denote the conditional prior distribution for $\left(\lambda_{1}, \lambda_{2}, \cdots, \lambda_{J}\right)$ without $\lambda_{k}$. We therefore derive $\pi\left(\lambda_{k} \mid \boldsymbol{\beta}, \boldsymbol{\omega}, \kappa^{-1}, D\right)$ as

$$
\begin{aligned}
& \lambda_{k}^{d_{k}} \exp \left\{-\lambda_{k} \omega_{i} \exp \left(\mathbf{x}_{\mathbf{i j j}}^{\prime} \boldsymbol{\beta}\right) \times\left[\sum_{(i, j) \in R_{k}}\left(s_{k}-s_{k-1}\right)+\sum_{(i, j) \in D_{k}}\left(t_{i j}-s_{k-1}\right)\right]\right\} \\
& \quad \times \pi\left(\lambda_{k} \mid \lambda^{(-k)}\right)
\end{aligned}
$$

\begin{tabular}{|c|c|c|c|c|c|c|c|c|c|c|c|c|}
\hline \multirow[t]{3}{*}{ Distance $(\mathrm{km})$} & \multicolumn{12}{|c|}{ Degradation (mm) } \\
\hline & \multicolumn{6}{|c|}{ Bogie I } & \multicolumn{6}{|c|}{ Bogie II } \\
\hline & 1 & 2 & 3 & 4 & 5 & 6 & 7 & 8 & 9 & 10 & 11 & 12 \\
\hline 33366 & 10.96 & 11.02 & 10.45 & 10.54 & 10.11 & 10.04 & 8.25 & 8.12 & l & 1 & 10.06 & 10.03 \\
\hline 87721 & 24.59 & 24.56 & 25.11 & 25.3 & 26.68 & 26.65 & 28.02 & 27.99 & 27.92 & 28.36 & 28.05 & 28.07 \\
\hline 161346 & 44.93 & 45.16 & 44.59 & 44.56 & 44.63 & 44.62 & 45.94 & 45.89 & 45.96 & 45.91 & 45.98 & 45.96 \\
\hline 204349 & 75.35 & 75.12 & 74.94 & 75.02 & 74.7 & 74.68 & 80.66 & 80.76 & 80.52 & 80.68 & 80.87 & 80.91 \\
\hline
\end{tabular}

Table 5.1

Degradation data of Locomotive 1.

Table 5.2

Degradation data of Locomotive 2.

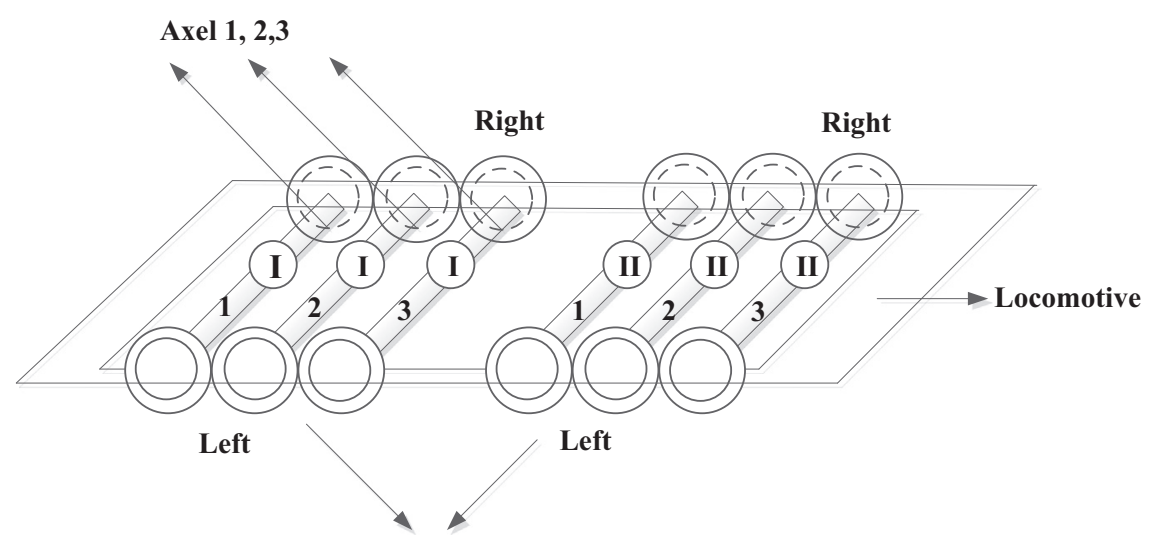

Bogie I, II

Fig. 5.1. Wheel positions specified in this study. 


\section{A general data-driven framework}

Instead of arguing the advantages and disadvantages of the two approaches (i.e., classical and Bayesian semi-parameter), in this paper, we propose the results from different models can complement each other. Following discussions in Sections 2 and 3, we now propose a general data-driven framework, using both classical and Bayesian degradation approaches to support the optimisation of PM strategies. As shown in Fig. 4.1, the framework is composed of a continuous improvement process, including four stages divided into nine sequential steps.

- Step 1: Collect degradation data. These include the observed values of a physical process.

- Step 2: Select degradation path. To determine the path, select from among several candidates for the studied system/units (for

Table 5.3

Statistics on lifetime data.

\begin{tabular}{|c|c|c|c|c|c|c|c|c|c|}
\hline \multirow[t]{2}{*}{ No. } & \multicolumn{2}{|c|}{ Positions } & \multicolumn{2}{|l|}{ Lifetime** } & \multirow[t]{2}{*}{ No. } & \multicolumn{2}{|c|}{ Positions } & \multicolumn{2}{|l|}{ Lifetime ${ }^{* *}$} \\
\hline & Loco. & Bogie & Exponential & Power & & Loco. & Bogie & Exponential & Power \\
\hline 1 & 1 & I & 316 & 334 & 13 & 2 & I & 230 & 316 \\
\hline 2 & 1 & I & 316 & 334 & 14 & 2 & I & 230 & 317 \\
\hline 3 & 1 & I & 314 & 331 & 15 & 2 & I & 230 & 312 \\
\hline 4 & 1 & I & 314 & 331 & 16 & 2 & I & 230 & 312 \\
\hline 5 & 1 & I & 316 & 334 & 17 & 2 & I & 229 & 305 \\
\hline 6 & 1 & I & 316 & 334 & 18 & 2 & I & 228 & 305 \\
\hline 7 & 1 & II & $291^{*}$ & $314^{*}$ & 19 & 2 & II & 218 & 269 \\
\hline 8 & 1 & II & $291^{*}$ & $314^{*}$ & 20 & 2 & II & 217 & 268 \\
\hline 9 & 1 & II & $289^{*}$ & $310^{*}$ & 21 & 2 & II & 237 & 273 \\
\hline 10 & 1 & II & $289^{*}$ & $310^{*}$ & 22 & 2 & II & 237 & 274 \\
\hline 11 & 1 & II & 312 & 329 & 23 & 2 & II & 222 & 284 \\
\hline 12 & 1 & II & 312 & 328 & 24 & 2 & II & 222 & 284 \\
\hline
\end{tabular}

* Right-censored data.

*** $\times 1000 \mathrm{~km}$

Probability - Weibull

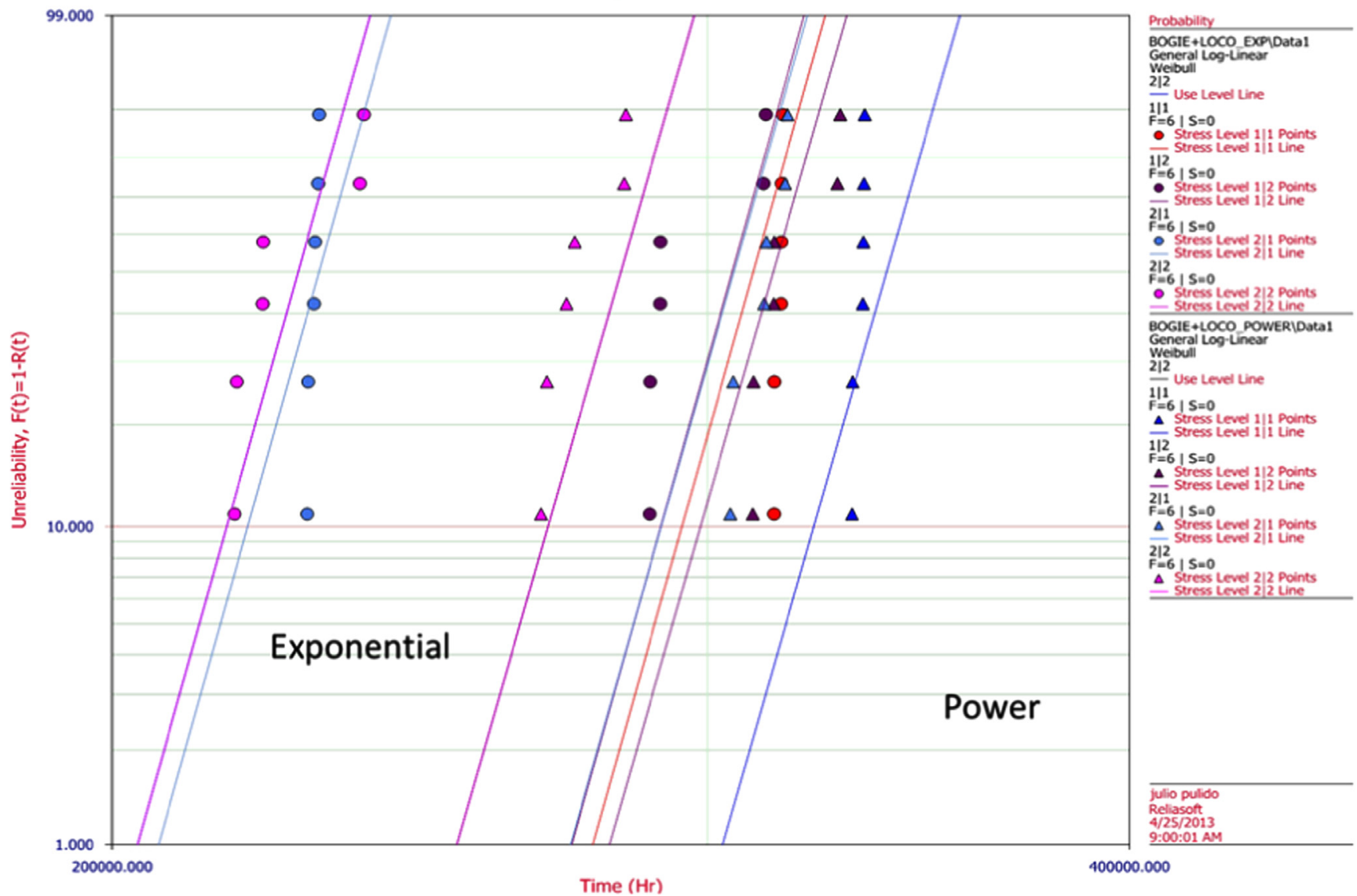

Fig. 5.2. Life data analysis. 
instance, linear degradation, Exponential degradation, Power degradation, Logarithmic degradation, Gompertz degradation, etc.).

- Step 3: Predict and get lifetime data. This step uses the results achieved in step 2 to predict the lifetime data. Note: data may be subject to uncertainties, such as imprecise measurement, censoring, truncated information, and interpretation errors.

- Step 4 and Step 5: Modelling. These steps consider reliability models using classical and Bayesian approaches (parametric or non-parametric), separately. The input data come from Step 3, and parameter configurations consider the physical process. The implementation has been discussed in Sections 2 and 3.

- Step 6: Compare results. Here, we check whether the results from different approaches are consistent. If they are, we move on to the next step; if they are not, we return to steps 4 and 5 and re-determine the model selection (or parameter configurations).

- Step 7: Make inferences. After achieving the acceptable results, we can perform reliability inference to determine system (or unit) reliability, find the failure distribution, etc. Note: results from different approaches can be used to complement each other.

- Step 8: Support PM strategy making. Based on the results from reliability analysis in step 7, PM strategies will be supported.

- Step 9: Update data and improve inference. Along with the passage of time, new data can be obtained, relegating "previous" inference results to "historical data". By updating "degradation data" and restarting at step 1, we can improve the reliability inference and optimise the PM strategies.
In summary, with this step-by-step method, we can create a continuous improvement process for the degradation inference and PM strategy making.

You will recall that the process has four stages. Steps 1, 2, and 3 are assigned to "stage 1 " when data for model implementation are prepared. Steps 4 and 5 are both assigned to "stage 2", where the classical and Bayesian approaches are carried out. Steps 6 to 8 are treated as "stage 3"; at this time, the results are checked and compared; in addition, knowledge is accumulated and PM strategies are improved upon by implementing various candidate reliability models. "Stage 4" consists only of step 9; at this point, a continuously improved loop can be obtained. In other words, by implementing the step-by-step procedure, we can accumulate and gradually update degradation knowledge. Equally, the reliability results will be improved upon and become increasingly robust, thereby improving the accuracy of the PM inference results.

\section{Case study}

We now consider a case study of the wheel-sets on two locomotives in a heavy haul cargo train to illustrate how to use the data-driven framework and the classical and Bayesian semiparametric approaches to support a company in preventative maintenance decision making.

Section 5.1 refers to data collection in the proposed framework (step 1). The degradation path and the lifetime data of the

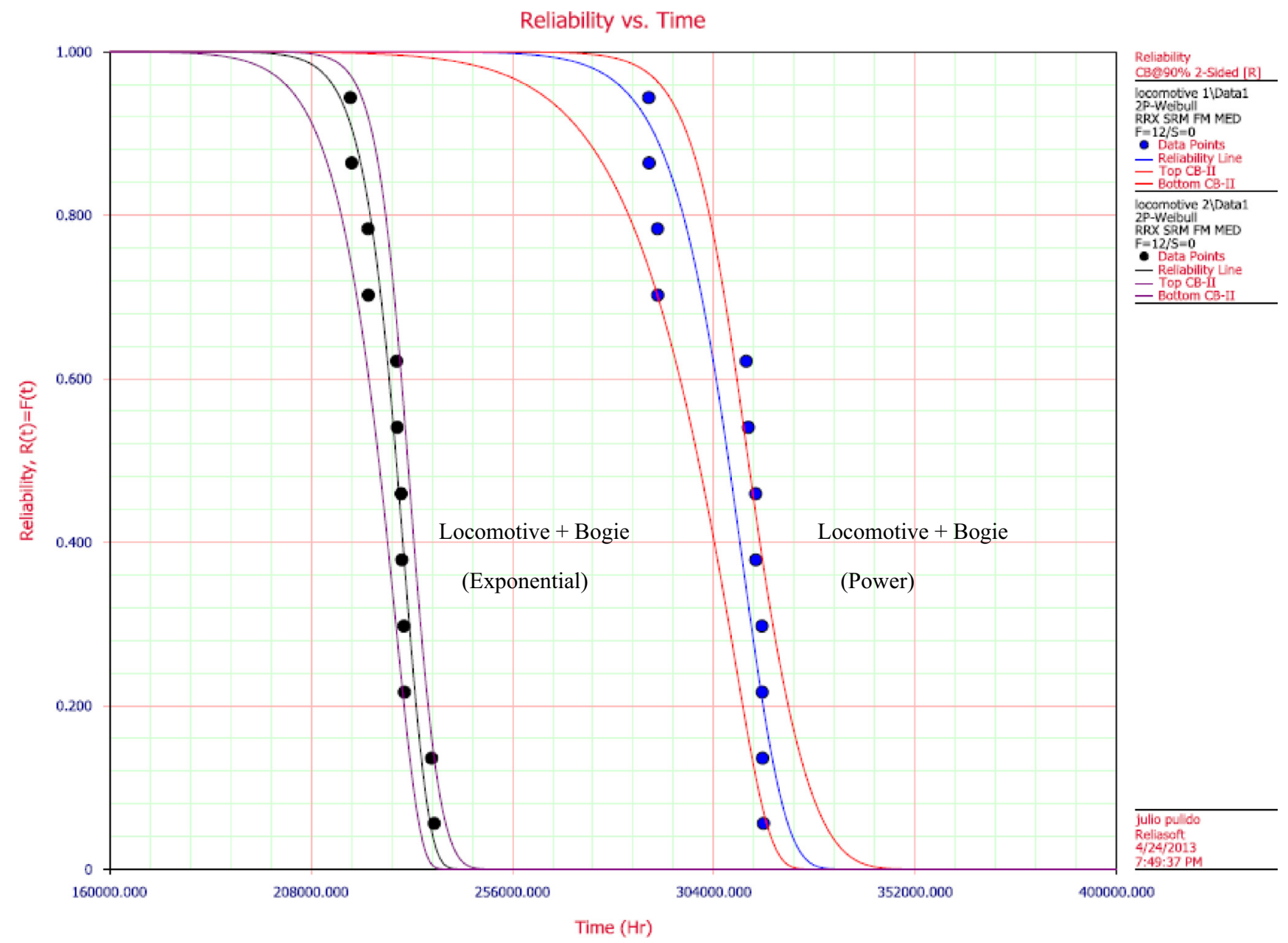

Fig. 5.3. Reliability curve for degradation type. 
locomotive wheel-sets are studied in Section 5.2 (steps 2 and 3). Sections 5.3 and 5.4 present results and discussions of the classical approach (step 4) and the Bayesian semi-parametric approach (step 5), respectively. As the results are not in conflict (step 6), we make reliability inferences (step 7) and suggest PM strategies (step 8 ). We conclude in Section 5.5 by presenting more comparisons of the classical and Bayesian semi-parametric approaches.

\subsection{Degradation data}

The data were collected by a Swedish company from November 2010 to January 2012 (see Tables 5.1 and 5.2). They comprise degradation data from two heavy haul cargo trains' locomotives (denoted as Locomotive 1 and Locomotive 2). Accordingly, there are two studied groups, and $n=2$. For each locomotive, see Fig. 5.1, there are two bogies (Bogie I, Bogie II), and each bogie has six wheel-sets, making a total of 12 wheels for each locomotive.

The diameter of a new locomotive wheel is $1250 \mathrm{~mm}$. In the company's current maintenance strategy, the wheel-set's diameter is measured after running a certain distance; note that this is not a data-driven approach. If it is reduced to $1150 \mathrm{~mm}$, the wheel-set is replaced by a new one. Otherwise, it is re-profiled or other maintenance strategies are implemented. Therefore, a threshold level for failure, denoted $a s l_{0}$, is defined as $100 \mathrm{~mm}\left(l_{0}=1250 \mathrm{~mm}-\right.$ $1150 \mathrm{~mm}$ ). The wheel-set's failure condition is assumed to be reached if the diameter reaches $l_{0}$. Tables 5.1 and 5.2 present the degradation data for the wheel-sets of Locomotive 1 and Locomotive 2, separately.

\subsection{Degradation path and lifetime data}

From the dataset, we can obtain 3 to 5 measurements of the diameter of each wheel during its lifetime. By connecting these measurements, we can determine a degradation trend. In their analyses of train wheel-sets, most studies $[11,16]$ assume a linear degradation path. In our study, considering both the results from degradation analysis in Weibull ++ and the type of physics of failure associated with wear and fatigue, we select Exponential and Power degradation models. An Exponential model is described by the following function (5.1) and the Power model by the subsequent function (5.2):

Exponential : $y=b \times e^{a \times x}$

Power : $y=b \times x^{a \times c}$

whereyrepresents the performance (here, it represents the diameters of the wheel-sets), $x$ represents time (here, it represents the running distance of the wheel-sets), and $a, b$ and $c$ are model parameters to be solved. Following the above discussion, we set $l_{0}=y$. The lifetimes for these wheels are now easily determined and are shown in Table 5.3. As discussed by Lin et al. [16], some lifetime data can be viewed as right-censored (denoted by asterisk in Table 5.3).

\subsection{Results and discussions from classical models}

As shown in Figs. 5.2 and 5.3, the exponential function for this set of data yields more conservative results and is in line with field

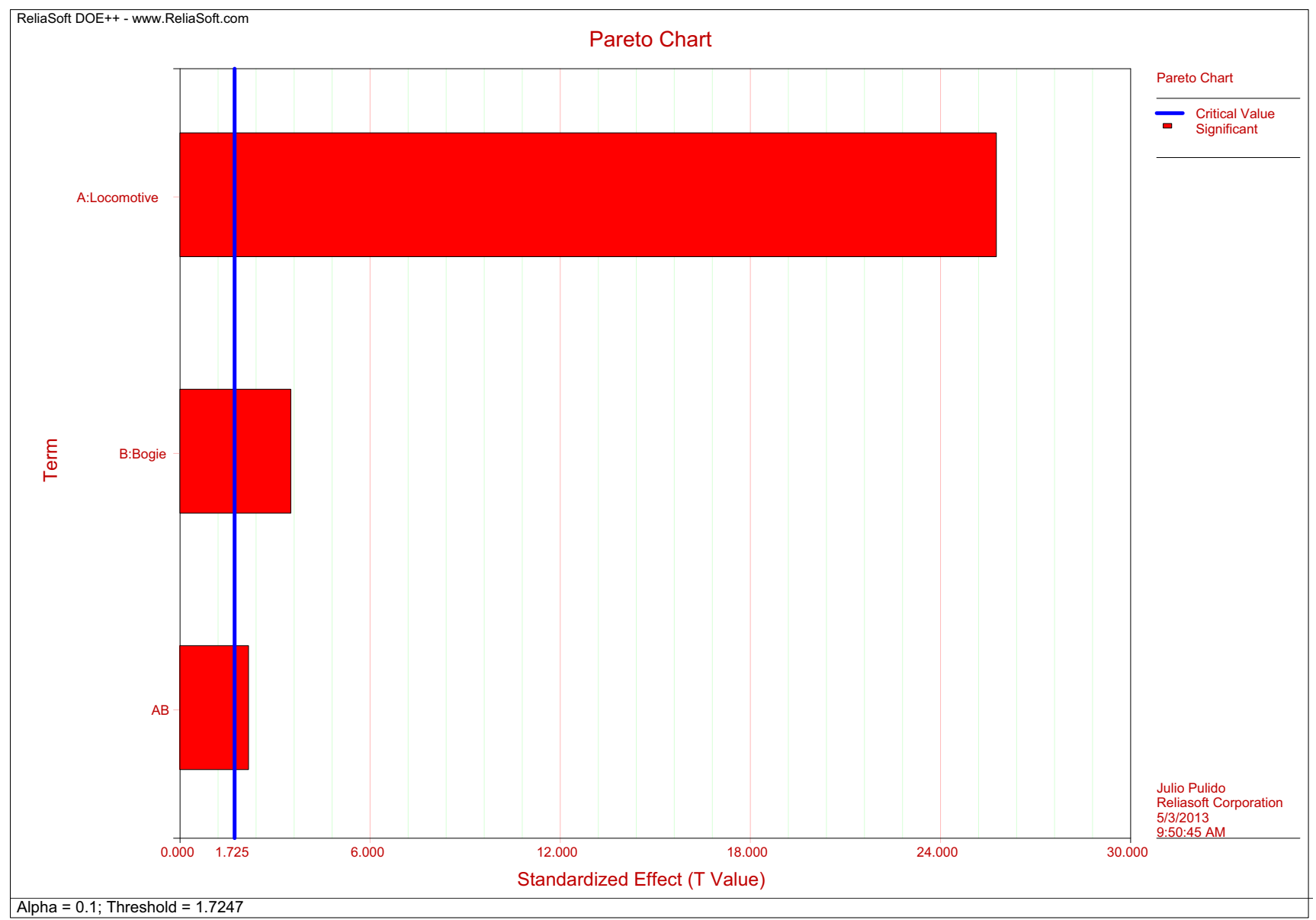

Fig. 5.4. Factors Pareto chart. 
observations when life data are compared at different stress levels as previously defined. Fig. 5.3 shows reliability values for Locomotive 2 and Bogie 2; both sides have 95\% confidence level.

Using the exponential degradation model, we perform a two factor full factorial DOE analysis and find that the locomotive, bogie and interaction are critical factors (see Fig. 5.4).

A review of the life stress relationship between the factors indicates the locomotive is a higher contributor to the degradation of the system than the bogie (Figs. 5.5 and 5.6).

Based on the analysis, we can reach the following conclusions. Independent of the Degradation model, the locomotive is the more critical stressor, as shown in the data above. Failure modes obtained from the data are similar for the locomotive and the bogies. Of the two stress conditions, level 2 is the highest for the locomotive and bogie, as shown in Fig. 5.6. Figs. 5.6 and 5.7 show the reliability values at each operating distance. Fig. 5.7 shows that Locomotive 2 has the highest degradation per distance travelled.

\subsection{Results and discussions of Bayesian semi-parametric models}

\subsubsection{Parameter configuration}

In this model, considering the wheels' positions specified in Section 5.1, the installed positions of the wheel-sets on a particular locomotive are specified by the bogie number and are defined as covariatesx. The covariates' coefficients are represented by $\boldsymbol{\beta}$. More specifically, $x=1$ represents the wheels mounted in Bogie I, while $x=2$ represents the wheels mounted in Bogie II. $\beta_{1}$ is the coefficient, and $\beta_{0}$ is defined as natural variability.

It is clear that a very small $k$ will make the model nonparametric. However, if $k$ is too small, estimates of the baseline hazard rate will be unstable, and if kis too large, a poor model fit could result. In our study, determining the degradation path requires us to make 3 to 5 measurements for each locomotive wheel; in other words, the lifetime data are based on the data acquired at 3 to 5 different inspections. Following the reasoning above, we divide the time axis into 6 sections piecewise. In our case study, no predicted lifetime exceeds $360000 \mathrm{~km}$. Therefore, $k=6$, and each interval is equal to $60000 \mathrm{~km}$. We get 6 intervals $(0,60000$ ), (60000, $12000] \ldots(300000,360000]$.

For convenience, we let $\lambda_{k}=\exp \left(b_{k}\right)$, and vague prior distributions are adopted as the following:

- Gamma frailty prior: $\omega_{i} \sim G a\left(\kappa^{-1}, \kappa^{-1}\right)$

- Normal prior distribution: $b_{k} \sim N\left(b_{k-1}, \kappa\right)$

- Normal prior distribution: $b_{1} \sim N(0, \kappa)$

- Gamma prior distribution: $\kappa \sim G a(0.0001,0.0001)$

- Normal prior distribution: $\beta_{0} \sim N(0.0,0.001)$

- Normal prior distribution: $\beta_{1} \sim N(0.0,0.001)$

At this point, the MCMC calculations are implemented with the software WinBUGS [27]. A burn-in of 10001 samples is used, with an additional 10000 Gibbs samples.

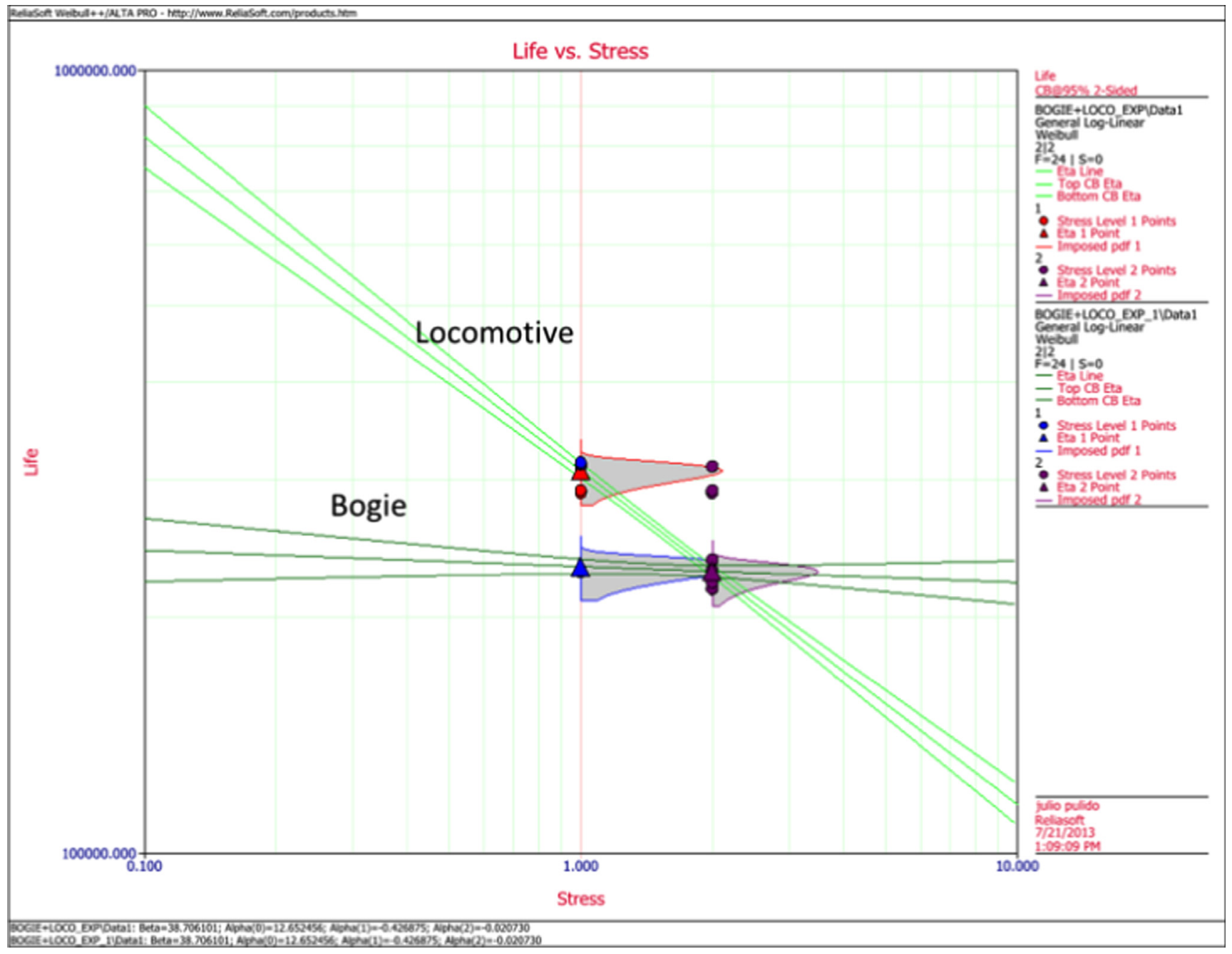

Fig. 5.5. Life vs. stress. 


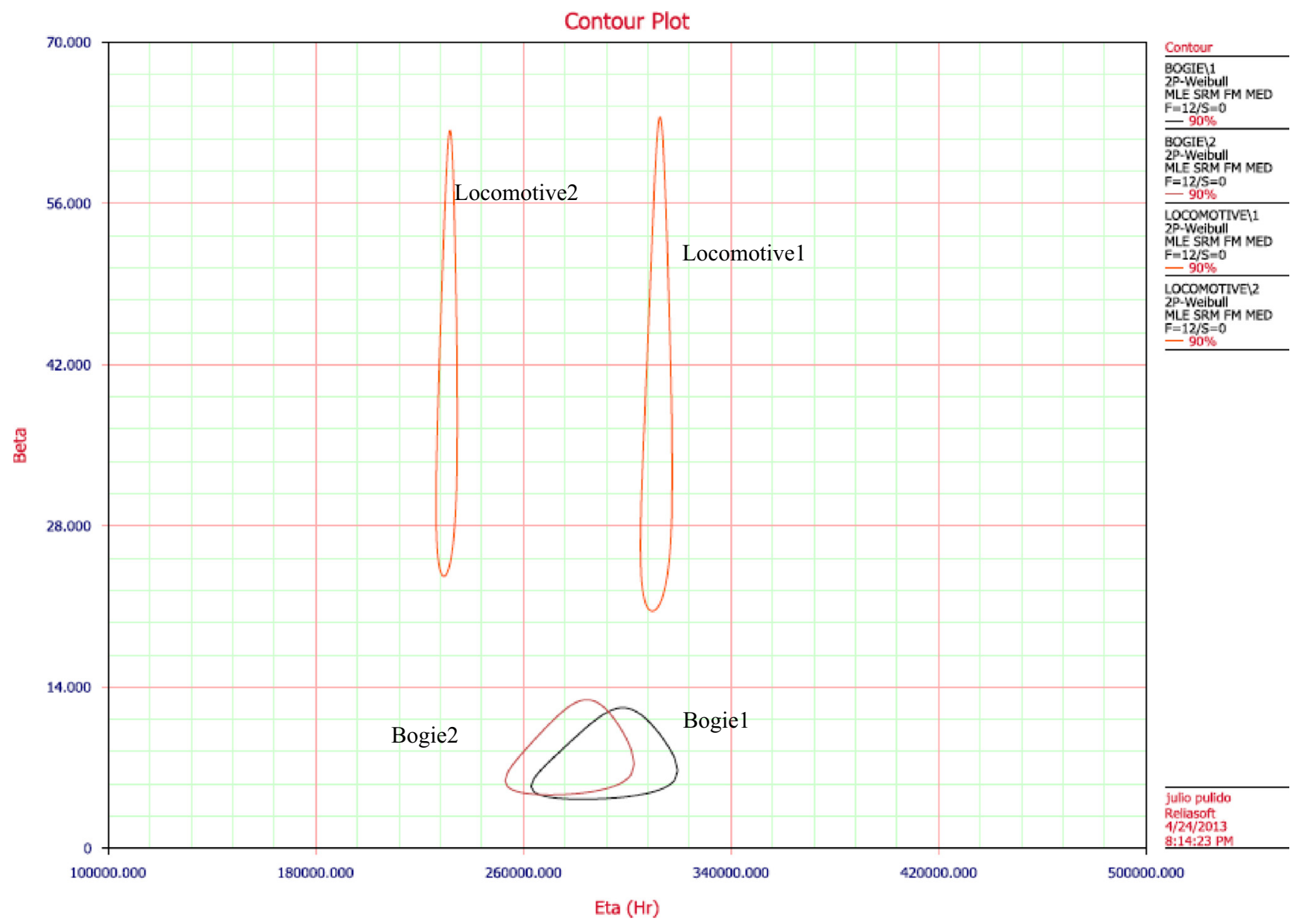

Fig. 5.6. Contour plot.

\subsubsection{Results from Bayesian semi-parametric model}

Following the convergence diagnostics, we consider the following posterior distribution summaries (Table 5.4): the parameters' posterior distribution mean, SD, MC error, and the 95\% highest posterior distribution density (HPD) interval.

In Table $5.4, \beta_{1}>0$ means wheels mounted in the first bogie (as $x=1$ ) have a shorter lifetime than those in the second (as $x=2$ ). However, the influence could possibly be reduced as more data are obtained in the future, because the 95\% HPD interval includes 0 point. In addition, the small value of $\beta_{1}(\approx 0.045)$ indicates that, in this case, heterogeneity among wheels installed in different bogies exists but is not significant. Because $\kappa<0.5$, heterogeneity among the locomotives does exist but is not significant either. However, the frailty factors obviously exist. For instance, $\omega_{1}<1$ suggests the predicted lifetimes for those wheels mounted on the first locomotive are longer than if the frailties are not considered; meanwhile, $\omega_{2}>1$ indicates the wheels mounted on the second locomotive have a shorter lifetime than if the frailties are not considered.

Baseline hazard rate statistics based on the above results $\left(b_{1}, \ldots, b_{6}\right)$ are shown in Table 5.5 and Fig. 5.8. At the fourth piecewise interval, the wheels' baseline hazard rate increases dramatically (1481.78). It is interesting that at the fifth piecewise interval, it decreases (185.49) but increases again after the sixth piecewise (22697.27).

By considering the random effects resulting from the natural variability (explained by covariates) and from the unobserved random effects within the same group (explained by frailties), we can determine other reliability characteristics of the lifetime distribution. The statistics on reliability $R(t)$ and cumulative hazard rate $\Lambda(t)$ for the two wheels mounted in different bogies are listed in Table 5.6, Figs. 5.9 and 5.10.

For Locomotive 1 and Locomotive 2, Figs. 5.9 and 5.10 show the plots of reliability and cumulative hazard, respectively. It should be pointed out that both Figs. 5.9 and 5.10 show change points in the wheel-sets. For example, the reliability declines sharply at the fourth and the sixth piecewise interval. Meanwhile, after the fifth and the sixth piecewise interval, the cumulative hazard increases dramatically.

\subsubsection{Discussions from Bayesian semi-parametric model}

The above results (Section 5.4.2) can be applied to maintenance optimisation, including wheel-set re-profiling optimisation, lifetime prediction and replacement optimisation, and preventive maintenance optimisation.

Before continuing, in Table 5.7, we list the re-profiling times (running distance/kilometres) for Locomotive 1 and Locomotive 2 , separately (in rows 1 and 3). We can see the difference between reprofiling polices: for Locomotive 1, re-profiling is done, at most, 5 times, whilst the wheels on Locomotive 2 are re-profiled, at most, 4 times. For greater clarification, we list them under the $k$ intervals. For instance, for Locomotive 1, the first re-profiling was performed at $106000 \mathrm{~km}$, placing it into the second piecewise interval. We can denote $\Delta D$ as the gap from the "current re-profiling" to the next one in each piecewise interval (rows 2 and 4). More specifically, for Locomotive 1, the first re-profiling is at $106000 \mathrm{~km}$, and the next at $144000 \mathrm{~km}$, creating a gap of $38000 \mathrm{~km}$ (=144000-106 000). For the last re-profiling, we use the boundary of $360000 \mathrm{~km}$ as the "next re-profiling". By comparing $\Delta D$, we can see the running 


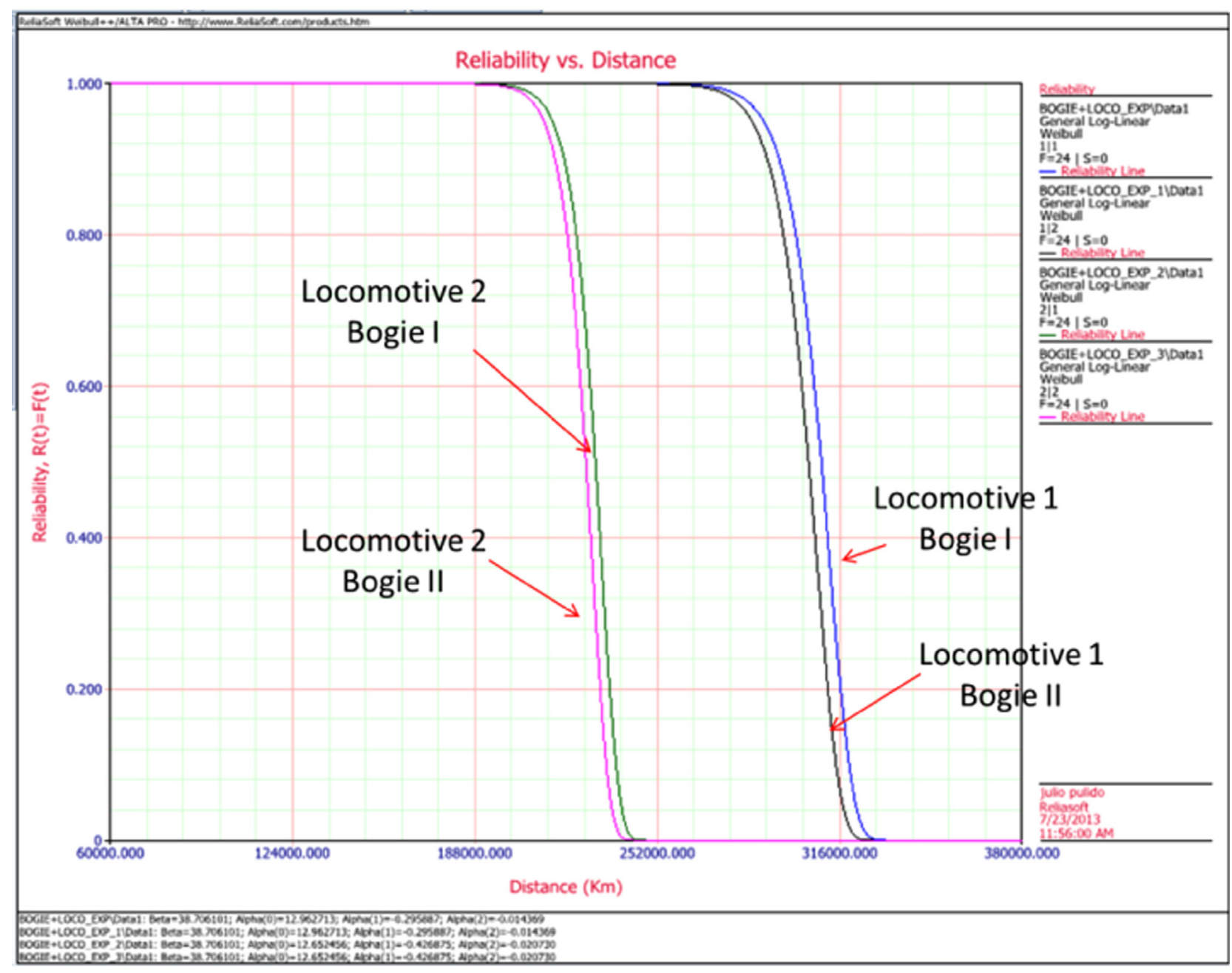

Fig. 5.7. Reliability curves at each condition.

Table 5.4

Posterior distribution summaries.

\begin{tabular}{lclll}
\hline Parameter & mean & SD & MC error & 95\% HPD interval \\
\hline$\beta_{0}$ & -12.08 & 4.184 & 0.4019 & $(-22.17,-4.802)$ \\
$\beta_{1}$ & 0.04517 & 0.4889 & 0.02025 & $(-0.948,0.9669)$ \\
$\kappa$ & 0.1857 & 0.1667 & 0.008398 & $(0.008616,0.6128)$ \\
$\omega_{1}$ & 0.5246 & 0.2878 & 0.01401 & $(0.06489,1.064)$ \\
$\omega_{2}$ & 1.473 & 0.5807 & 0.01596 & $(0.6917,2.948)$ \\
$b_{1}$ & -0.3764 & 4.113 & 0.1619 & $(-8.316,5.933)$ \\
$b_{2}$ & 0.3571 & 4.95 & 0.2429 & $(-8.836,8.181)$ \\
$b_{3}$ & 2.272 & 4.61 & 0.3029 & $(-6.4,10.81)$ \\
$b_{4}$ & 7.301 & 4.106 & 0.3938 & $(0.2106,17.13)$ \\
$b_{5}$ & 5.223 & 4.225 & 0.3281 & $(-3.166,13.41)$ \\
$b_{6}$ & 10.03 & 3.993 & 0.3802 & $(2.72,19.3)$ \\
\hline
\end{tabular}

Table 5.5

Baseline hazard rate statistics.

\begin{tabular}{lllllll}
\hline $\begin{array}{l}\text { Piecewise intervals } \\
(\times \mathbf{1 0 0 0} \mathbf{~ k m})\end{array}$ & $\mathbf{1}$ & $\mathbf{2}$ & $\mathbf{3}$ & $\mathbf{4}$ & $\mathbf{5}$ & $\mathbf{6}$ \\
\cline { 2 - 7 } & $\mathbf{( 0 ,}$ & $\mathbf{( 6 0}$, & $\mathbf{( 1 2 0}$, & $\mathbf{( 1 8 0 ,}$ & $(\mathbf{2 4 0}$, & $\mathbf{( 3 0 0 ,}$ \\
& $\mathbf{6 0 ]}$ & $\mathbf{1 2 0}]$ & $\mathbf{1 8 0}]$ & $\mathbf{2 4 0}]$ & $\mathbf{3 0 0 ]}$ & $\mathbf{3 6 0}]$ \\
\hline$\lambda_{k}$ & 0.069 & 1.43 & 9.7 & 1481.78 & 185.49 & 22697.27 \\
\hline
\end{tabular}

distances of the wheels between profiling. If we do not consider the first interval's statistics (normally, the new wheel is treated as running in a good condition), the largest values appear at the fourth

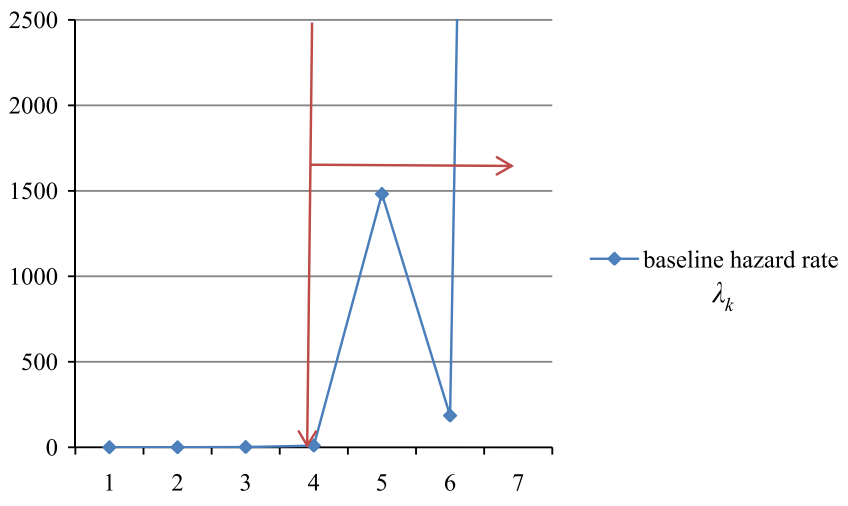

Fig. 5.8. Plot of baseline hazard rate.

interval for each locomotive, consistent with the findings from Figs. 5.8 and 5.9. Therefore, the re-profiling time will influence the wheel-set degradation rate. If re-profiling is performed earlier than $272000 \mathrm{~km}$ for Locomotive 1, the degradation rate could be reduced, as could the baseline hazard rate. Meanwhile, the reliability in piecewise interval 4 could be increased. This conclusion could also explain why at the fifth interval, the baseline hazard rate decreases while the reliability increases. As discussed above, we recommend improving the re-profiling polices by considering the re-profiling intervals. 
Table 5.6

Reliability and cumulative hazard statistics.

\begin{tabular}{|c|c|c|c|c|c|c|c|c|}
\hline \multirow[t]{3}{*}{ Distance $(1000$ km) } & \multicolumn{4}{|c|}{ Reliability $R(t)$} & \multicolumn{4}{|c|}{ Cumulative hazard $\Lambda(t)$} \\
\hline & \multicolumn{2}{|c|}{ Locomotive 1} & \multicolumn{2}{|c|}{ Locomotive 2} & \multicolumn{2}{|c|}{ Locomotive 1} & \multicolumn{2}{|c|}{ Locomotive 2} \\
\hline & Bogie I & Bogie II & Bogie I & Bogie II & Bogie I & Bogie II & Bogie I & Bogie II \\
\hline 60 & 0.999872 & 0.999866 & 0.99964 & 0.999624 & $5.57 \mathrm{E}-05$ & $5.82 \mathrm{E}-05$ & 0.000156 & 0.000164 \\
\hline 120 & 0.999466 & 0.999442 & 0.998502 & 0.998433 & 0.000232 & 0.000243 & 0.000651 & 0.000681 \\
\hline 180 & 0.99458 & 0.994331 & 0.984857 & 0.984162 & 0.00236 & 0.002469 & 0.006627 & 0.006933 \\
\hline 240 & 0.330536 & 0.314054 & 0.044672 & 0.038695 & 0.480781 & 0.502996 & 1.349964 & 1.41234 \\
\hline 300 & 0.840949 & 0.834245 & 0.614843 & 0.601179 & 0.07523 & 0.078707 & 0.211236 & 0.220996 \\
\hline 360 & $8.98 \mathrm{E}-12$ & $2.77 \mathrm{E}-12$ & $9.61 \mathrm{E}-32$ & $3.54 \mathrm{E}-33$ & 11.0466 & 11.55701 & 31.01723 & 32.4504 \\
\hline
\end{tabular}

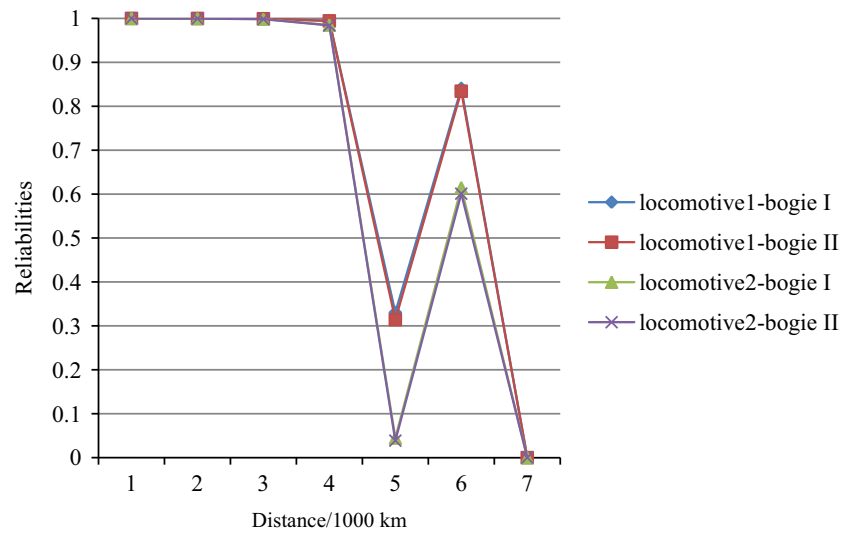

Fig. 5.9. Plot of the reliabilities for Locomotive 1 and Locomotive 2.

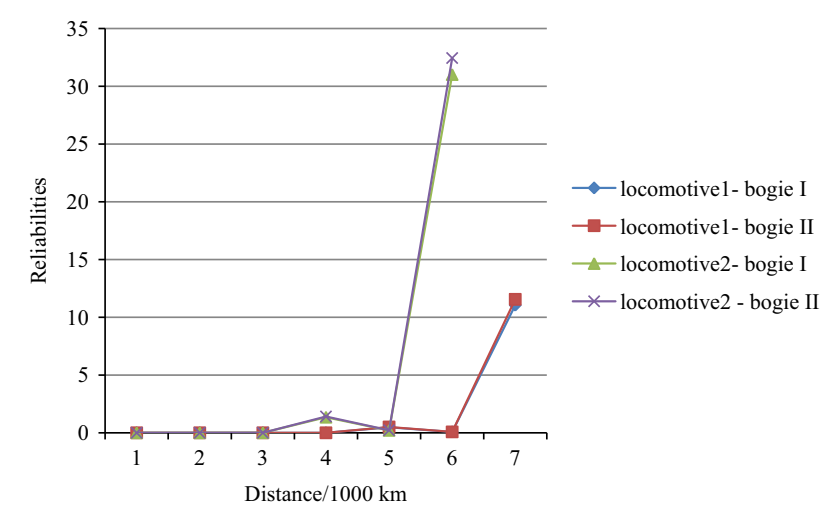

Fig. 5.10. Plot of the cumulative hazard for Locomotive 1 and Locomotive 2 .

Table 5.7

Re-profiling statistics.

\begin{tabular}{|c|c|c|c|c|c|c|c|}
\hline No. & $\begin{array}{l}\text { Piecewise } \\
\text { intervals* }\end{array}$ & 1 & 2 & 3 & 4 & 5 & 6 \\
\hline & Re-profiling & $\begin{array}{l}(0, \\
60]\end{array}$ & $\begin{array}{l}(60, \\
120]\end{array}$ & $\begin{array}{l}(120, \\
180]\end{array}$ & $\begin{array}{l}(180, \\
240]\end{array}$ & $\begin{array}{l}(240, \\
300]\end{array}$ & $\begin{array}{l}(300, \\
360]\end{array}$ \\
\hline 1 & Locomotive 1 & 0 & 106 & 144 & 191 & 272 & 309 \\
\hline 2 & $\Delta D_{1}$ & 106 & 38 & 47 & 81 & 37 & 51 \\
\hline 3 & Locomotive 2 & 33 & 87 & 161 & 204 & 0 & 0 \\
\hline 4 & $\Delta D_{2}$ & 54 & 74 & 43 & 189 & 1 & 1 \\
\hline
\end{tabular}

Now consider seasonal influence (in this case, temperature). In this case, re-profiling at the fourth piecewise was done between March 2010 and September 2010. Although the degradation rate should be lower than if it were winter, if the time between reprofiling is too long, the baseline hazard rate could increase dramatically and the reliability could decrease. Again, we recommend improving the re-profiling polices by considering the reprofiling intervals, although seasonal influence should also be included.

Interestingly, in Figs. 5.8-5.10, the change points appearing in the fourth piecewise interval (from 180000 to $360000 \mathrm{~km}$ ) indicate that after running about $180000 \mathrm{~km}$, the locomotive wheel has a high risk of failure. Although the $\Delta D$ is sometimes larger (for instance, $\Delta D_{1}$ equals 106 at the first interval), it is more stable before the fourth piecewise interval. Rolling contact fatigue (RCF) problems could start at the fourth interval (after $180000 \mathrm{~km}$ ). Therefore, we recognize the whole period as two stages: one is stable (before $180000 \mathrm{~km}$ ), and the second is unstable. Special attention should be paid if the wheels have run longer than these change points (in other words, reaching an unstable stage). In addition, because reprofiling may leave cracks over time and reduce the wheel's lifetime, we recommend cracks be checked after re-profiling to improve the lifetime.

Although the difference is not that obvious, the wheel-sets installed in the first bogie should be given more attention during maintenance. Especially when the wheel-sets are re-profiled, they should be checked, starting with the first bogie to avoid duplication of effort. Note that in the studied company, the wheel-set inspecting sequences are random; this means the first checked wheel could belong in the second bogie. After the second checked wheel is lathed or re-profiled, if the diameter is less than predicted, the first checked wheel-set might need to be lathed or re-profiled again. Therefore, starting with the wheel-set installed in the first bogie could improve maintenance effectiveness.

Determining reliability characteristics distributed over a wheelset's lifetime (see Table 5.6) could be used to optimise replacement strategies. The results could also support related predictions for spares inventory.

Last but not least, the different frailties between locomotives could be caused by the different operating environments (e.g., climate, topography, and track geometry), configuration of the suspension, status of the bogies or spring systems, operation speeds, the applied loads and human influences (such as drivers' operations, maintenance policies and actions of the lathe operator). Specific operating conditions should be considered when designing maintenance strategies because even if the locomotives and wheel types are the same, the lifetimes and operating performance could differ.

\subsection{Comparison of models}

For the sake of comparison, Table 5.8 presents the reliability statistics using the classical model and an Exponential degradation path, as discussed in Section 2.

The results of the two approaches show Locomotive 2 has lower reliability than Locomotive 1 . In addition, for both 
Table 5.8

Reliability statistics using classical model.

\begin{tabular}{|c|c|c|c|c|}
\hline \multirow[t]{3}{*}{ Distance $(1000$ km) } & \multicolumn{4}{|l|}{ Reliability $R(t)$} \\
\hline & \multicolumn{2}{|l|}{ Locomotive 1} & \multicolumn{2}{|l|}{ Locomotive 2} \\
\hline & Bogie I & Bogie II & Bogie I & Bogie II \\
\hline 60 & 1.000000000000 & 1.000000000000 & 1.000000000000 & 1.000000000000 \\
\hline 120 & 1.000000000000 & 1.000000000000 & 1.000000000000 & 1.000000000000 \\
\hline 180 & 0.999999999500 & 0.999999999100 & 0.999949988500 & 0.999912782200 \\
\hline 240 & 0.999963596200 & 0.999936513100 & 0.032469287900 & 0.002535299700 \\
\hline 300 & 0.814489640600 & 0.699174645800 & 0.000000000000 & 0.000000000000 \\
\hline 360 & 0.000000000000 & 0.000000000000 & 0.000000000000 & 0.000000000000 \\
\hline
\end{tabular}

Locomotive 1 and Locomotive 2, before the fourth piecewise interval, the reliability statistics from the classical approach have a higher value; after the fifth piecewise interval, the reliability statistics from the Bayesian approach have a higher value.

In addition, considering the application process and results achieved by the different approaches in the case study, we can make the following comparisons of classical and Bayesian approaches:

- First, the Bayesian semi-parametric degradation approach needs less hypothesis than classical methods because the piecewise constant hazard regression model is more flexible.

- Second, the dependence within subgroups can be considered an unknown and unobservable risk factor of the gamma frailty model; fewer assumptions are required when implementing the Bayesian approach.

- Third, the use of prior information from different sources will improve the precision of the predictions, reflecting the superiority of Bayesian approach.

- Fourth, with the Bayesian semi-parametric approach, changepoints are more obvious and easily studied.

- Fifth, classical approaches are more reliable as the dataset is large, but the Bayesian approach is dominant because the dataset is smaller. That being said, the dataset is large enough, so the choices made for PM strategies depend on preference or accepted risk level.

- Sixth, the Bayesian approach is more complex and not as easily manipulated by engineers; this complexity includes parameter configuration, MCMC implementation, etc.

- Seventh, the outputs from the classical approach are more familiar to applicators and, therefore, are more intuitive and more easily interpreted and analysed.

In summary, using either approach has its advantages and disadvantages. Following the above discussion, we recommend comparing the results achieved by different approaches, accept them based on specified risk level when designing PM strategies, and use them to complement each other under specified conditions (for instance, a particular decision maker's preference).

\section{Conclusions}

This paper proposes a reliability study based on a general datadriven framework using both classical and Bayesian semi-parametric degradation approaches to illustrate how degradation data can be modelled and analysed to flexibly determine reliability during preventive maintenance strategy making. The case study considers both an Exponential and a Power degradation path for the wheel-sets of a locomotive and concludes the former is the better option. Using a classical approach, it uses both accelerated life tests (ALT) and design of experiments (DOE) technology to determine how each critical factor, i.e, locomotive and bogie, affects the prediction of performance. Within the Bayesian semi-parametric approach, the piecewise constant hazard rate is used to establish the distribution of the wheel-set lifetime. The gamma shared frailties $\omega_{i}$ are used to explore the influence of unobserved covariates within the same locomotive. By introducing covariate $\mathbf{x}_{i}$ 's linear function $\mathbf{x}_{\mathrm{ij}}^{\prime} \boldsymbol{\beta}$, the influence of the bogie in which a wheel is installed can be taken into account. The MCMC technique is used to integrate high-dimensional probability distributions to make inferences and predictions about model parameters. The results from the classical and Bayesian semi-parametric approaches can complement each other.

The results of the case study suggest the lifetime of wheel-sets can differ depending on where they are installed (in which bogie they are mounted) on the locomotive. The gamma frailties help with exploring the unobserved covariates and, thus, they improve the model's precision. We can determine wheel-set reliability characteristics, including the baseline hazard rate $\lambda(t)$, reliability $R(t)$, and cumulative hazard rate $\Lambda(t)$. The results also indicate the existence of change points. As Figs. 5.8-5.10 show, wheel-set reliability can be divided into two stages: stable and unstable at $180000 \mathrm{~km}$. The results allow us to evaluate and optimise wheel replacement and maintenance strategies (including the reprofiling interval, inspection interval, lubrication interval, depth and optimal sequence of re-profiling, and so on).

The proposed data-driven framework in this paper can be applied to cargo train wheel-sets or to other technical problems (e.g. other industries, other components).

\section{Acknowledgements}

The authors would like to thank the editor and anonymous referees for their constructive comments. The authors thank Luleå Railway Research Centre (Järnvägstekniskt Centrum, Sweden) and Swedish Transport Administration (Trafikverket) for initiating the research study and providing financial support (JVTC projectnr:274). We also thank Richard House for his support in the review and development of this paper. Finally, we thank Thomas Nordmark, Ove Salmonsson and Hans-Erik Fredriksson at LKAB for their support and their discussions of the locomotive wheels.

\section{References}

[1] Bernasconi A, et al. An integrated approach to rolling contact sub-surface fatigue assessment of railway wheels. J Wear 2005;258:973-80.

[2] Braghin F, et al. A mathematical model to predict railway wheel profile evolution due to wear. J Wear 2006;261:1253-64.

[3] Briano E., et.al. Design of experiment and Monte Carlo simulation as support for gas turbine power plant availabilty estimation. In: Proceedings of the 12th WSEAS international conference on AUTOMATIC CONTROL, MODELLING \& SIMULATION. Conference proceedings. World Scientific and Engineering Academy and Society (WSEAS) Stevens Point, Wisconsin, USA. 2010: 223-230. 
[4] Clayton DG. A model for association in bivariate life tales and its application in epi-demiological studies of familial tendency in chronic disease incidence. Biometicka 1978;65:141-51.

[5] Clayton P. Tribological aspects of wheel-rail contact: a review of recent experimental research. J Wear 1996;191:170-83.

[6] Craiu RV, Lee TCM. Model selection for the competing-risks model with and without masking. J Tech 2005;47(4):457-67.

[7] Demarqui FN, Loschi RH, Dey DK, Colosimo EA. A class of dynamic piecewise exponential models with random time grid. J Stat Plann Inf 2012:142:728-42.

[8] Donato $P$, et al. Design and signal processing of a magnetic sensor array for train wheel detection. J Sens Actuators A 2006;132:516-25.

[9] Doostparast M, Kolahan F, Doostparast M. A reliability-based approach to optimize preventive maintenance scheduling for coherent systems. J Reliab Eng Syst Saf 2014;126:98-106.

[10] Douglas C. Montgomery design and analysis of experiments. 8th ed. Wiley; 2012.

[11] Freitas MA, et al. Using degradation data to assess reliability: a case study on train wheel degradation. J Qual Reliab Eng Int 2009;25:607-29.

[12] Goulart A, Zhan W. A design of experiment (DOE) analysis of the performance of uplink real-time traffic over a $3 G$ network. In: IEEE international conference on wireless \& mobile computing, networking \& communication. Conference proceedings 2008. http://dx.doi.org/10.1109/WiMob.2008.110.

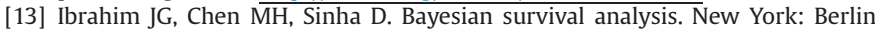
Heidelberg; 2001.

[14] Johansson A. Andersson C. Out-of-round railway wheels-a study of whee polygonalization through simulation of three-dimensional wheel-rail interaction and wear. J Vehicle Syst Dyn 2005;43(8):539-59.

[15] Levin MA, Kalal TT. Improving product reliability. John Wiley; 2003.

[16] Lin J, Asplund M, Parida A. Reliability analysis for degradation of locomotive wheels using parametric Bayesian approach. J Qual Reliab Eng Int 2014;30:657-67.

[17] Liu YM, et al. Multiaxial fatigue reliability analysis of railroad wheels. J Reliab Eng Syst Saf 2008;93:456-67.

[18] Meeker WQ, Escobar LA. Statistical methods for reliability data. Wiley; 1998.

[19] Moghaddam KS, Usher JS. Sensitivity analysis and comparison of algorithms in preventive maintenance and replacement scheduling optimization models. J Comput Ind Eng 2011;61:64-75.

[20] Musharraf M, et al. A virtual experimental technique for data collection for a Bayesian network approach to human reliability analysis. J Reliab Eng Syst Saf 2014;132:1-8

[21] Palo M. Condition-based maintenance for effective and efficient rolling stock capacity assurance: a study on heavy haul transport in Sweden. Doctoral thesis. Luleå University of Technology, Sweden; 2014

[22] Pombo J, Ambrosio J, Pereira M. A railway wheel wear prediction tool based on a multibody software. J Theor Appl Mech 2010;48(3):751-70.

[23] ReliaSoft DOE + + Experiment design and analysis reference. ReliaSoft Corporation; 2008

[24] Sahu SK, Dey DK, Aslanidou H, Sinha D. A Weibull regression model with gamma frailties for multivariate survival data. J Lifetime Data Anal 1997;3:123-37.

[25] Skarlatos D, Karakasis K, Trochidis A. Railway wheel fault diagnosis using a fuzzy-logic method. J Appl Acoust 2004:65:951-66.

[26] Soderholm P. A system view of the no fault found (NFF) phenomenon. J Reliab Eng Syst Saf 2007;92:1-14.
[27] Spiegelhalter D., et al.. WinBUGS User Manual (Version 1.4). January; 2003. 〈http://www.mrc-bsu.cam.ac.uk/bugs〉.

[28] Stratman B, Liu Y, Mahadevan S. Structural health monitoring of railroad wheels using wheel impact load detectors. J Fail Anal Prevent 2007;7 (3):218-25.

29] Tassini N, et al. A numerical model of twin disc test arrangement for the evaluation of railway wheel wear prediction methods. J Wear 2010;268:660-7.

[30] Wienke A. Frailty models in survival analysis. PhD thesis; 2007.

[31] Yang C, Letourneau S. Learning to predict train wheel failures. In: Conference proceedings. The 11th ACM SIGKDD international conference on knowledge discovery and data mining (KDD 2005). Chicago, Illinois, USA.

[32] Yuan T, et al. Bayesian analysis for accelerated life tests using a Dirichlet process Weibull mixture model. J IEEE Trans Reliab 2014;63:58-67.

[33] Zhang X, Kang J, Jing T. Degradation modeling and maintenance decisions based on Bayesian belief networks. J IEEE Trans Reliab 2014;63:620-33.

Dr. Jing Lin is currently an Assistant Professor in the Division of Operation and Maintenance Engineering, at Luleå University of Technology (LTU), Sweden. She obtained her PhD degree in Management from Nanjing University of Science and Technology (NJUST), China, in April 2008. After completing her doctorate, she worked 3 years for SKF Co., Ltd as an Asset Management Consultant. Dr. Lin's research interests primarily lie in RAMS and Asset Management. She has published $50+$ peer reviewed journal and conference papers.

Dr. Julio Pulido is a Sr. Director of Design for Reliability Engineering at ReliaSoft Corporation. His responsibility is to drive the capability development of Design for Reliability for consulting clients. He holds a BS from the Federal University of Bahia, Brazil, an MS from the Federal University of Rio Grande do Sul, Brazil, a PhD from the Federal University of Rio de Janeiro, Brazil, PhD Research at Duke University and an MBA from Xavier University and a Certificate in Information Technology Management from the University of Chicago. His specialty is in the area of Structural Analysis, Design for vibration and Structural Reliability, and Accelerated Testing Techniques. Dr. Pulido has $19+$ years of experience in leading Design Assurance Organizations. He has published over 50 works at different internationa symposiums.

Matthias Asplund is a PhD student in the Division of Operation and Maintenance Engineering, at Luleå University of Technology (LTU), Sweden, since 2011. His research area is RAMS with railway topics in focus. He has twelve years working experience from product development, lean production, maintenance and railway engineering. He got his Master degree in Mechanical Engineering with focus on Applied Mechanics from Luleå Technical University. His last work before studies to PhD was Track Engineering for the Swedish Infrastructure Management. 